\title{
Prevalence and determinants of virological failure, genetic diversity and drug resistance among people living with HIV in a minority area in China: a population- based study
}

Dan Yuan ${ }^{1 \dagger}$, Meijing Liư ${ }^{2 \dagger}$, Peng Jia ${ }^{3,4}$, Yiping Li ${ }^{1}$, Yuling Huang ${ }^{1}$, Li Ye ${ }^{1}$, Laze Api ${ }^{5}$, Maogang Chen ${ }^{6}$, Liang Yao ${ }^{7}$, Zixin Wang ${ }^{8}$, Honglu Liu', Shu Liang ${ }^{1 *}$ and Shujuan Yang ${ }^{2,4^{*}}$ (D)

\begin{abstract}
Background: Liangshan Yi Autonomous Prefecture is one of the areas that most severely affected by human immunodeficiency virus (HIV) in China, and virological failure on antiretroviral therapy (ART) is serious in this area. Analyses of prevalence and determinants of ART failure, the genetic diversity and drug resistance among people living with HIV (PLWH) helps improve HIV treatment efficiency and prevent HIV transmission.

Methods: A total of 5157 PLWH were recruited from 2016 to 2017. The venous blood samples were subjected to RT-PCR, followed by sequencing of the HIV-1 pol gene, targeting the protease and reverse transcriptase fragments. HIV-1 diversity was analyzed using the DNAStar software and drug resistance mutations were analyzed using the Stanford University HIV Drug Resistance Database.

(Continued on next page)
\end{abstract}

\footnotetext{
* Correspondence: liangshu523@163.com; rekiny@126.com

${ }^{\dagger}$ Dan Yuan and Meijing Liu contributed equally to this work.

${ }^{1}$ Center for AIDS/STD Control and Prevention, Sichuan Center for Disease Control and Prevention, Chengdu, China

${ }^{2}$ West China School of Public Health and West China Fourth Hospital, Sichuan University, Chengdu, Sichuan, China

Full list of author information is available at the end of the article
}

(C) The Author(s). 2020 Open Access This article is licensed under a Creative Commons Attribution 4.0 International License, which permits use, sharing, adaptation, distribution and reproduction in any medium or format, as long as you give appropriate credit to the original author(s) and the source, provide a link to the Creative Commons licence, and indicate if changes were made. The images or other third party material in this article are included in the article's Creative Commons licence, unless indicated otherwise in a credit line to the material. If material is not included in the article's Creative Commons licence and your intended use is not permitted by statutory regulation or exceeds the permitted use, you will need to obtain permission directly from the copyright holder. To view a copy of this licence, visit http://creativecommons.org/licenses/by/4.0/ The Creative Commons Public Domain Dedication waiver (http://creativecommons.org/publicdomain/zero/1.0/) applies to the data made available in this article, unless otherwise stated in a credit line to the data. 


\begin{abstract}
(Continued from previous page)
Results: A total of 2156 (41.81\%) PLWH showed virological failure on ART. Males (ORm =1.25), heterosexual behaviors and drug injection $(\mathrm{ORm}=1.44)$ and mother to child transmission routes $(\mathrm{ORm}=1.58)$, the clinical stage of AIDS (ORm = 1.35), having used illicit drugs and shared the needles ( $1-4$ times: ORm = 1.34; more than 5 times: $\mathrm{ORm}=1.52)$, having ever replaced $A R T$ regimen $(\mathrm{ORm}=1.48)$ increased the risk of virological failure among PLWH, while higher education lever $(\mathrm{ORm}=0.77)$ and $\geq 12$ months on ART $(12 \sim 36$ months: ORm $=0.72 ; \geq 36$ months: $\mathrm{ORm}=0.66$ ) was associated with lower likelihood of virological failure. The data revealed that CRF07_BC (1508, 95.62\%) were the most common strains, and the drug-resistant rate was $32.10 \%$ among PLWH with virological failure in this area. The high frequencies of drug resistance were found in EFV and NVP of NNRTIS, ABC, FTC and 3TC of NRTIs, and TPV/r in PIs. The most common mutations in NNRTIs, NRTIs and PIs were K103N/KN (64.69\%), M184V/MV/I (36.29\%) and Q58E/QE (4.93\%), respectively.

Conclusion: We concluded that surveillance of virological failure, HIV-1 subtypes, and drug resistance to understand HIV-1 epidemiology and guide modification of ART guidelines, and target prevention and control strategies should be formatted to reduce the virological failure and drug resistance to promote viral suppression and prevent HIV-1 transmission.
\end{abstract}

Keywords: HIV, Antiretroviral therapy, Virological failure, HIV-1 subtypes, Drug resistance

\section{Background}

Human immunodeficiency virus (HIV) infections in humans affect more than 37 million individuals worldwide, and approximately 0.85 million people living with HIV (PLWH) live in China. The number of PLWH has increased by 14\% from 2016 to 2017 [1]. Liangshan Yi Autonomous Prefecture, as the largest traditional settlement of Yi minority people in China, is the area affected most severely by HIV in China [2]. By the end of 2018, there have been 38,545 PLWH reported in Liangshan, and prevalence rates of HIV in five counties were the highest in China [3, 4]. Effective HIV interventions should be formatted to reduce HIV transmission in this area.

Antiretroviral therapy (ART) is highly effective in suppressing HIV, stopping the progression of HIV disease and reducing the risk of HIV transmissions [5, 6]. The rapid scale-up of ART could lead to a significant decline in morbidity and mortality among PLWH, which has been confirmed by many studies $[5,7,8]$. ART is a longterm treatment with the potential for drug toxicity and the emergence of HIV resistance, setting the stage for a suboptimal response or lack of sustained response to therapy that is defined as virological failure. Unfortunately, virological failure not only compromises therapeutic effects for individuals but also endangers the population as a whole [9]. In Liangshan, the virological failure rate is reported to be about $36-45 \%[10,11]$, which is more serious than average in China [12], mainly due to the poor adherence to ART and inadequate drug levels. The high prevalence of virological failure may lead to a large extent of genotypic HIV-1 drug resistance. When an individual is infected with drug resistance mutations, the resistant strain may become the dominant strain for further transmission of HIV-1 drug resistance [13], and consequently increasing the number of antiretroviral-naive patients and reducing their therapeutic effectiveness [14]. Understanding the risk factors of virological failure could help to target HIV prevention and intervention strategies [15].

$\mathrm{HIV}$ is characterized by its great genetic variability and responsibility for developing drug-resistant mutations with high viral replication rates. This not only increases the risk of virological failure on antiretroviral treatment, but also results in the spread of drug-resistant strains, which brings a huge challenge to controlling the HIV epidemic and preventing the HIV spread. The genotype of HIV-1 is related to the route of transmission, and there are differences in epidemic scale and distribution characteristics $[16,17]$. The HIV-1 genotype distribution had been described in parts of China $[18,19]$. However, little data is known about that in the highest HIV epidemic area of China. Therefore, we aimed to analyze prevalence and determinants of ART failure, and understand the genetic diversity and drug resistance among PLWH, which could help us better understand HIV epidemiology and allow a timely modification of ART guidelines in this area.

\section{Methods}

\section{Study participants}

Participants were recruited through a multi-stage cluster sampling strategy. The county with the most severe HIV epidemic was selected from all 17 counties in Liangshan Prefecture. According to the basic information system for Acquired Immune Deficiency Syndrome (AIDS) prevention and treatment, all PLWH in this county were included in our study. We have tried to identify as many PLWH as possible through all three possible channels: (1) Provider-Initiated HIV Testing \& Counseling (PITC) service for HIV, (2) Voluntary Counseling and Testing (VCT) 
service through fixed VCT sites and non-government organizations (NGOs), (3) HIV sentinel surveillance (HSS) for unmarried youths, pregnant women, community population, and drug users. The inclusion criteria for PLWH were: (1) receiving confirmatory HIV diagnosis, (2) being permanent residents or had stayed in Liangshan area for more than 5 years, (3) receiving ART in the local township health center for more than 6 months. The exclusion criteria for participants were having have major psychiatric illness (e.g., schizophrenia and bipolar disorder.

According to the inclusion criteria and exclusion criteria, medical staff phoned candidate participants about the study and confirmed their eligibility to join the study. All participants signed informed consent forms before enrollment. Finally, a total of 5157 PLWH were included into the study.

\section{Data collection}

Questionnaire was used to ask all participants in private rooms to collected the socio-demographic information, possible route of HIV transmission, HIV-related behaviors and disease-related characteristics. The sociodemographic information including gender, age, occupation, current marital status, ethnicity, education level. The route of HIV transmission included drug injection, heterosexual behaviors and drug injection, casual sexual behavior and risk behaviors related to mother-to-child transmission treatment time. The HIV-related behaviors included information about needle sharing, number of casual sexual partners and history of sexually transmitted disease except for HIV. The disease related characteristics were extracted from their medical record, including viral load, stage of the disease, the initial regimen and time of ART and change of ART regimen. The above information was collected from National AIDS reporting system. When a newly diagnosed with HIV-1 infection was found or received ART, the detailed information (the initial regimen and time of ART) would be collected and inputted into system.

\section{Laboratory tests}

Each participant was asked to provide $5 \mathrm{~mL}$ of venous blood for detecting the viral load of HIV-1. The plasma samples were isolated from each participant, and preserved in $\mathrm{a}-80^{\circ} \mathrm{C}$ freezer until analysis. HIV viral load was measured in the Sichuan Center for Disease Control and Prevention. Virological failure in ART was defined as HIV RNA level $\geq 1000$ copies/ml $[20,21]$ after receiving ART for more than 6 months.

\section{Nucleic acid extraction, amplification, sequencing and drug-resistance analyses}

Total viral nucleic acid was extracted from 200- $\mu$ l plasma using an automatic extraction machine (MagNA Pure LC
2.0 system, Roche, Branchburg, NJ, USA). The full-length protease gene in $\mathrm{Pol}$ region and the first 300 codons of the reverse transcriptase gene was amplified by using reverse transcription polymerase chain reaction (RT-PCR). The amplified products were purified in and sequenced at Beijing Genomics Research Center Ltd., in China.

The HIV-1 pol sequences obtained in the study, together with reference sequences of different subtypes and CRFs, were edited and aligned using ChromasProl.33, and the sequence alignments were manually performed by BIOEDIT Sequence Alignment Editor software (Ibis Biosciences, Carlsbad, CA, USA). The detailed amplification, sequencing and drug resistance analyses were detailed described previously $[22,23]$.

\section{Statistical analysis}

Data were analyzed using SPSS version 21.0 for Windows (SPSS, Inc., Chicago, IL, USA). Categorical variables were expressed as frequencies and percentages and compared using the chi-squared $\left(x^{2}\right)$ test. Using the presence of virological failure in ART as the dependent variable, univariate odds ratios $(\mathrm{ORu})$ and $95 \%$ confidence interval (CI) for socio-demographic information, possible route of HIV transmission, HIV-related behaviors and disease-related characteristics were estimated. A summary model was obtained by fitting a multiple regression model that considered all variables that were significant in the respective univariate analysis as candidates. Multivariate odds ratios $(\mathrm{ORm})$ and respective 95\%CI were obtained. Was used for data analysis, with $p<0.05$ taken as statistically significant.

\section{Results}

Prevalence and determinants of virological failure on ART among PLWH

A total of 2156 (41.81\%) PLWH showed virological failure on ART from 2016 to 2017. Among PLWH with virological failure, majority of them were male, peasant, married, illiteracy, Yi minority, having HIV transmission route of drug injection, the first CD4+ counts of $200 \sim 500$ counts/ul, having never used illicit drugs and shared the needle, having no casual sexual partners and history of sexually transmitted diseases except for HIV (Table 1).

In univariate analysis, gender, age, occupation, current marital status, ethnicity, HIV transmission route, $\mathrm{CD} 4^{+}$ level, stage of disease, needle sharing and the number of casual sexual partners, the initial regimen of ART and change of ART regimen were significantly associated with risk of virological failure on ART $(p<0.05)$. In multivariate logistic regression model, we found that males, heterosexual behaviors and drug injection and mother to child transmission routes, stage of AIDS, having used illicit drugs and shared the needles, having ever 
Table 1 Prevalence and determinants of virological failure on ART among PLWH

\begin{tabular}{|c|c|c|c|c|}
\hline \multirow[t]{2}{*}{ Variables } & $V L \geq 1000$ copies/ml & \multirow{2}{*}{$\begin{array}{l}\text { Total } \\
\text { N }\end{array}$} & \multirow[t]{2}{*}{ ORu } & \multirow[t]{2}{*}{ ORm } \\
\hline & $\begin{array}{l}\text { Virologic failure } \\
n=2156 \text { (rate, } n / \text { N\%) }\end{array}$ & & & \\
\hline \multicolumn{5}{|l|}{ Socio-demographics } \\
\hline \multicolumn{5}{|l|}{ Gender } \\
\hline Female & $710(36.90)$ & 1925 & 1.0(ref) & 1.0 (ref) \\
\hline Male & $1446(44.70)$ & 3232 & $1.39(1.23-1.56)^{* * *}$ & $1.25(1.03-1.50)^{* * *}$ \\
\hline \multicolumn{5}{|l|}{ Age } \\
\hline$\leq 15$ & $172(48.86)$ & 352 & 1.0 (Ref.) & \\
\hline $15 \sim 40$ & $1423(41.45)$ & 3433 & $0.74(0.59-0.92) * * *$ & \\
\hline$>40$ & $533(40.94)$ & 1302 & $0.72(0.57-0.92) * * *$ & - \\
\hline Unknown & $28(40.00)$ & 70 & - & \\
\hline \multicolumn{5}{|l|}{ Occupation } \\
\hline Employed & $37(34.91)$ & 106 & 1.0 (Ref.) & \\
\hline Peasant & $1736(41.85)$ & 4148 & $1.34(0.90-2.01)$ & \\
\hline Students and children & $136(46.74)$ & 291 & $1.64(1.03-2.60)^{* *}$ & - \\
\hline Unknown & $247(40.36)$ & 612 & - & \\
\hline \multicolumn{5}{|l|}{ Current marital status } \\
\hline Married & $1406(41.80)$ & 3366 & 1.0 (Ref.) & \\
\hline Single & $428(45.77)$ & 934 & $1.18(1.02-1.36) * *$ & \\
\hline Widowed or Divorced & $99(35.87)$ & 276 & $0.78(0.60-1.01)^{*}$ & - \\
\hline Unknown & $223(38.38)$ & 581 & - & \\
\hline Ethnicity & $(0.00)$ & & & \\
\hline Yi & $2137(41.70)$ & 5125 & 1.0 (Ref.) & \\
\hline Han and others & $19(59.38)$ & 32 & $2.04(1.01-4.15)^{* *}$ & - \\
\hline \multicolumn{5}{|l|}{ Education level } \\
\hline Illiteracy & $1339(42.58)$ & 3145 & 1.0 (Ref.) & 1.0 (Ref.) \\
\hline Primary school & $581(40.77)$ & 1425 & $0.93(0.82-1.05)^{*}$ & $0.77(0.66-0.89)^{* *}$ \\
\hline Secondary or above & $70(38.67)$ & 181 & $0.85(0.63-1.16)$ & $0.69(0.46-1.04)$ \\
\hline Unknown & $166(40.89)$ & 406 & - & \\
\hline \multicolumn{5}{|l|}{ HIV transmission route } \\
\hline Heterosexual behaviors & $715(37.10)$ & 1927 & 1.0 (Ref.) & 1.0 (Ref.) \\
\hline Drug injection & $747(43.08)$ & 1734 & $1.28(1.12-1.47)^{* * *}$ & $1.12(0.93-1.35)$ \\
\hline Heterosexual behaviors and drug injection & $325(49.02)$ & 663 & $1.63(1.36-1.95) * * *$ & $1.44(1.15-1.82)^{* *}$ \\
\hline Mother to child & $163(48.95)$ & 333 & $1.63(1.29-2.05) * * *$ & $1.58(1.21-2.08)^{* *}$ \\
\hline Others and unknown & $206(41.20)$ & 500 & - & - \\
\hline \multicolumn{5}{|l|}{ The first $\mathrm{CD}^{+}$counts, counts/ul } \\
\hline$\leq 200$ & $346(46.82)$ & 739 & 1.0 (Ref.) & \\
\hline $200 \sim 500$ & $986(41.29)$ & 2388 & $0.80(0.68-0.94)^{* *}$ & \\
\hline$>500$ & $646(40.68)$ & 1588 & $0.78(0.65-0.93)^{* *}$ & - \\
\hline Unknown & $178(40.27)$ & 442 & - & \\
\hline \multicolumn{5}{|l|}{ Stage of disease } \\
\hline HIV & 784 (37.44) & 2094 & 1.0 (Ref.) & 1.0 (Ref.) \\
\hline AIDS & $985(44.49)$ & 2214 & $1.34(1.19-1.51)^{* * *}$ & $1.35(1.17-1.55)^{* * *}$ \\
\hline Unknown & $387(45.58)$ & 849 & - & \\
\hline
\end{tabular}


Table 1 Prevalence and determinants of virological failure on ART among PLWH (Continued)

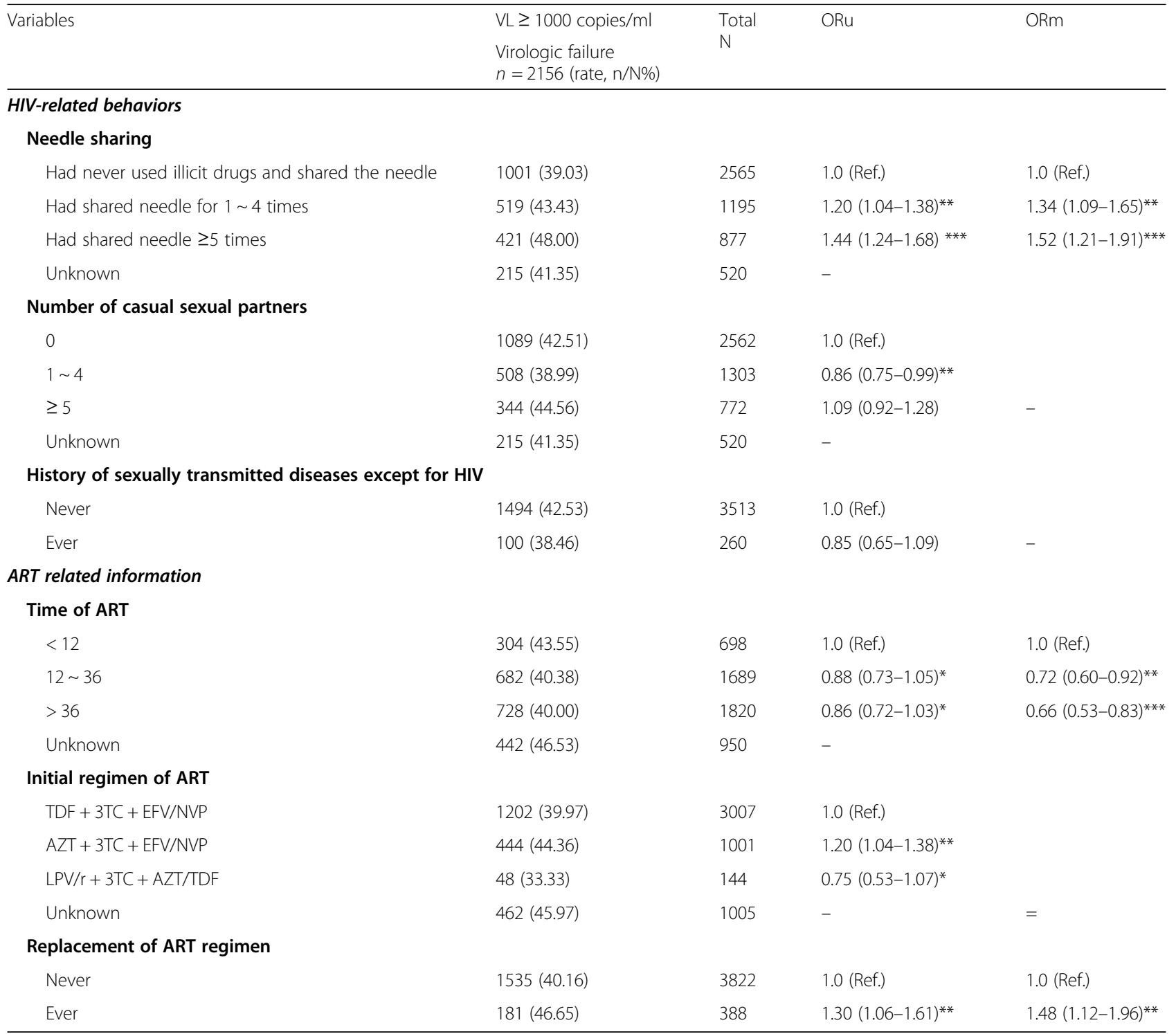

ORu univariate odds ratios, ORm multivariate odds ratios, Cl confidence interval, TDF tenofovir, 3TC lamivudine, NVP nevirapine, EFV efavirenz, AZT Zidovudine, $L P V / r$ fosamprenavir/ritonavir

Variables with $p<0.2$ in the univariate analysis as candidates were selected by a summary multiple logistic regression model

${ }^{*} P$ value $<0.2 ;{ }^{* *} P$ value $<0.05 ;{ }^{* *} P$ value $<0.001$

replaced ART regimen significantly increased the risk of virological failure among PLWH $(p<0.05)$, while higher education level and time on ART $\geq 12$ months were associated with lower likelihood of virological failure $(p<0.05)$ (Table 1$)$.

\section{Prevalence of drug resistance among PLWH with virological failure on ART}

Drug-resistant mutation in 1576 (73.10\%, 1576/2156) samples were successfully detected. The phylogenetic analyses based on the pol regions showed that CRF07. BC $(1508,95.62 \%)$ were the most common strains in this area, and followed by CRF08_BC (42, 2.66\%) and C (26,
$1.72 \%)$ subtypes were the most common strains in $\mathrm{Si}$ chuan province (Fig. 1).

Among PLWH with virological failure, the drugresistant rate was as high as $32.10 \%(507 / 1576)$. The prevalence of drug resistance was high in nonnucleoside reverse transcriptase inhibitors (NNRTIs) in all treatment regimens, then followed by nucleoside reverse transcriptase inhibitors (NRTIs) and protease inhibitors (PIs) (Table 2). The high frequencies of drug resistance were found in Efavirenz (EFV) and Nevirapine (NVP) of NNRTIs, Abacavir (ABC), Emtricitabine (FTC) and Lamivudine (3TC) of NRTIs, and tipranavir / ritonavir $(\mathrm{TPV} / \mathrm{r})$ in PIs. The $\mathrm{LPV} / \mathrm{r}+$ 


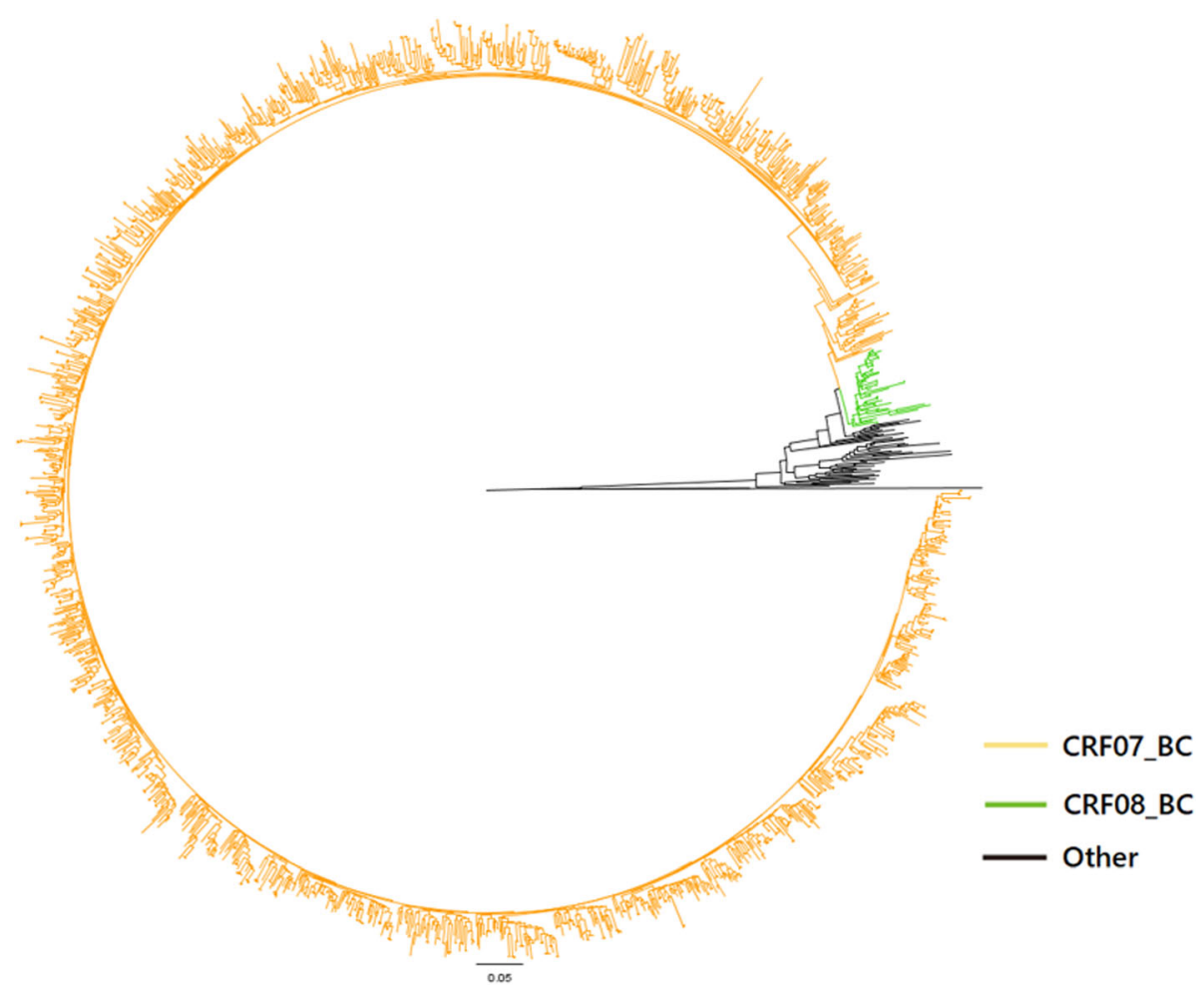

Fig. 1 Phylogenetic tree analyses of the HIV-1 pol sequences in PLWH with virological failure on ART; CRF07_BC accounted for 95.62\% of the HIV1 pol sequences; CRF08_BC accounted for 2.66\% of the HIV-1 pol sequences; Others were $C$ subtypes and reference strains

$3 \mathrm{TC}+\mathrm{AZT} / \mathrm{TDF}$ regimen showed the lowest drug resistance to PIs, NRTIs, and NNRTIs compared to the other two regimens.

\section{Antiretrovairal resistance mutations}

The drug-resistant mutation frequency to NNRTIs $(35.98 \%, 567 / 1576)$ was much higher than that to NRTIs (14.72\%,232/1576) and PIs (2.22\%,35/1576) among PLWH with virological failure. The most common mutations in NNRTIs were K103N/KN (64.69\%), V179D/E (23.47\%) and $\mathrm{Y} 181 \mathrm{C} / \mathrm{YC} / \mathrm{I}$ (14.00\%), they were M184V/MV/I (36.29\%), T215F/FS/TNSY (7.50\%) and K219Q (5.92\%) in NRTIs, and they were Q58E/QE (4.93\%), L10F/LFI (0.39\%) and M46L (0.39\%) in PIs (Fig. 2).

\section{Discussion}

The findings confirmed that the prevalence of virological failure on ART (41.81\%) among PLWH was high in this area. A high prevalence of subtype CRF07_BC (95.62\%) was observed among PLWH with virological failure on ART, which was different from other areas of China [24, 25]. Thus, it is a great challenge to successfully suppress HIV and decrease drug resistance in PLWH in this area.

Our study found that male PLWH and illiteracy were associated with a higher likelihood of incomplete viral suppression of virological failure on ART. Compared to females, males are more likely to discontinue from ART, which results in virological failure [26, 27]. Part of the reasons for this may be explained by (1) Compared to women, men generally have poorer healthcare-seeking behaviors, poorer ART uptake and a higher risk of immunotherapy failure observed in this study [28] (2) Women have a lower body mass index and are more likely to maintain a higher concentration of drugs in their bodies than men, so they are more likely to achieve viral suppression [29], (3) Men have more unhealthy behaviors such as smoking and drinking than women, which can lead to poorer adherence to medication and lower overall treatment success [30]. Illiteracy patients often lack sufficient understanding of the importance of ART and regular medication, as well as lack of communication skills with physicians [31,32], which may increase the risk of virological failure. Therefore, it is necessary to develop effective measures to promote drug compliance of ART targeting males PLWH and illiteracy in Liangshan.

We also found that patients who had been on ART $\leq 12$ months, and had changed ART regimens were more likely to experience virologic failure. Time on ART $\leq 12$ months was associated with a high risk of virological failure than time on ART $\geq 12$ months. This result was similar to a finding from a study in 
Table 2 Prevalence of drug resistance among PLWH with virological failure on ART

\begin{tabular}{|c|c|c|c|c|c|}
\hline & $\mathrm{TDF}+3 \mathrm{TC}+\mathrm{NVP} / \mathrm{EFV}$ & $\mathrm{AZT}+3 \mathrm{TC}+\mathrm{NVP} / \mathrm{EFV}$ & $\mathrm{LPV} / \mathrm{r}+3 \mathrm{TC}+\mathrm{AZT} / \mathrm{TDF}$ & Others & $\begin{array}{l}\text { Total } \\
N=1576(\%)\end{array}$ \\
\hline & $n=928(\%)$ & $n=318(\%)$ & $n=47(\%)$ & $N=283(\%)$ & $N=1576(\%)$ \\
\hline Pls & $13(1.40)$ & $9(2.83)$ & $1(2.13)$ & $6(2.12)$ & $35(2.22)$ \\
\hline ATV/r & - & - & - & $1(0.35)$ & $2(0.13)$ \\
\hline $\mathrm{DRV} / \mathrm{r}$ & - & - & - & - & - \\
\hline $\mathrm{FPV} / \mathrm{r}$ & - & $1(0.31)$ & - & $1(0.35)$ & $3(0.19)$ \\
\hline $\mathrm{IDV} / \mathrm{r}$ & - & - & - & $1(0.35)$ & $2(0.13)$ \\
\hline $\mathrm{LPV} / \mathrm{r}$ & - & - & - & $1(0.35)$ & $2(0.13)$ \\
\hline NFV & $1(0.11)$ & $1(0.31)$ & - & $2(0.71)$ & $6(0.38)$ \\
\hline $\mathrm{SQV} / \mathrm{r}$ & - & - & - & $1(0.35)$ & $2(0.13)$ \\
\hline $\mathrm{TPV} / \mathrm{r}$ & $12(1.29)$ & $8(2.52)$ & $1(2.13)$ & $5(1.77)$ & $31(1.97)$ \\
\hline NRTIs & 97 (10.45) & $55(17.30)$ & $2(4.26)$ & $39(13.78)$ & $232(14.72)$ \\
\hline$A B C$ & $92(9.91)$ & $55(17.30)$ & $2(4.26)$ & 39 (13.78) & $227(14.40)$ \\
\hline AZT & $26(2.80)$ & $22(6.92)$ & $2(4.26)$ & $8(2.83)$ & $66(4.19)$ \\
\hline D4T & $44(4.74)$ & $26(8.18)$ & $2(4.26)$ & $19(6.71)$ & $110(6.98)$ \\
\hline DDI & $45(4.85)$ & $25(7.86)$ & $2(4.26)$ & 19 (6.71) & $110(6.98)$ \\
\hline FTC & $91(9.81)$ & $55(17.30)$ & $2(4.26)$ & 39 (13.78) & $226(14.34)$ \\
\hline $3 T C$ & $91(9.81)$ & 55 (17.30) & $2(4.26)$ & 39 (13.78) & $226(14.34)$ \\
\hline TDF & $30(3.23)$ & $18(5.66)$ & $1(2.13)$ & $16(5.65)$ & $81(5.14)$ \\
\hline NNRTIs & 269 (28.99) & $108(33.96)$ & $10(21.28)$ & $90(31.80)$ & 567 (35.98) \\
\hline EFV & $261(28.13)$ & 103 (32.39) & $10(21.28)$ & 88 (31.10) & $550(34.90)$ \\
\hline ETR & 63 (6.79) & $42(13.21)$ & $1(2.13)$ & $24(8.48)$ & 154 (9.77) \\
\hline NVP & $261(28.13)$ & $105(33.02)$ & $10(21.28)$ & 88 (31.10) & $552(35.03)$ \\
\hline RPV & 96 (10.34) & $58(18.24)$ & $1(2.13)$ & 35 (12.37) & $225(14.28)$ \\
\hline
\end{tabular}

TDF Tenofovir, 3TC Lamivudine, NVP Nevirapine, EFV Efavirenz, AZT Zidovudine, LPV/r Fosamprenavir/ritonavir, ATV/r Atazanavir/ritonavir, DRV/r Darunavir/ritonavir, FPV/r Fosamprenavir/ritonavir, IDV/r Indinavir/Ritonavir, NFV Nelfinavir, SQV/r Ritonavir-boosted saquinavir, TPV/r Tipranavir/ritonavir, ABC Abacavir, D4T Stavudine, DDI Didanosine, FTC Emtricitabine, ETR Etravirine, RPV Rilpivirine

Zimbabwe, which showed that compare to time on ART $\geq 4$ years, patients whose time on ART $\leq 4$ years had more risk of treatment failure. Because the latter may have taken the wrong medicine or forgotten it due to lack of experience [30, 31]. Therefore, the current results all suggest the necessity of increasing the medication guidance, strengthening the management of treatment follow-up, and urging the patients to take medicine on time and following the prescribed amount [32]. Finally, the replacement of the ART regimen was also associated with virological failure. Patients who had replaced the ART regimen were more likely to experience treatment failure, possibly because they were not systematically tested for viral load and drug resistance before replacement [33]. However, our study didn't obtain the information on the reasons for the replacement of the ART regimen. Therefore, the capacity of doctors should be improved, and the process of changing the ART regimen should be standardized. Viral load and drug resistance detection should be required before the replacement of the ART regimen.
Our studies found that the subtype CRF07_BC (95.62\%) was most prevalent and was the predominant subtype, followed by CRF08_BC. CRF07_BC, which was one of the most prevalent CRFs in China [34-39] and was first identified in the intravenous drug use (IDU) population in Xinjiang in 1997 [38, 39]. CRF07_BC was first introduced into Yunnan and then spread into Sichuan and Gansu, and finally to Xinjiang along drug transporting routes [40,41]. Liangshan is located along the drug trafficking routes from the "Golden Triangle" to the northwest and central China, and injection drug use was the main infection risk factors of HIV infection and about $33.2-56.9 \%$ of the new HIV-1 patients were drug users [42], and it can be assumed that CFR07_BC strain is prevalent in this area. Given the changing profile of the HIV epidemic with the shift of high-risk behaviors from IDU to sexual contact, the heterosexual transmission has surpassed injective drug use and became the predominant route of HIV transmission in this area $[2,3]$. The dominant prevalent of CRF07_BC illustrated that infected IDUs are the main source of transmission to other populations. 


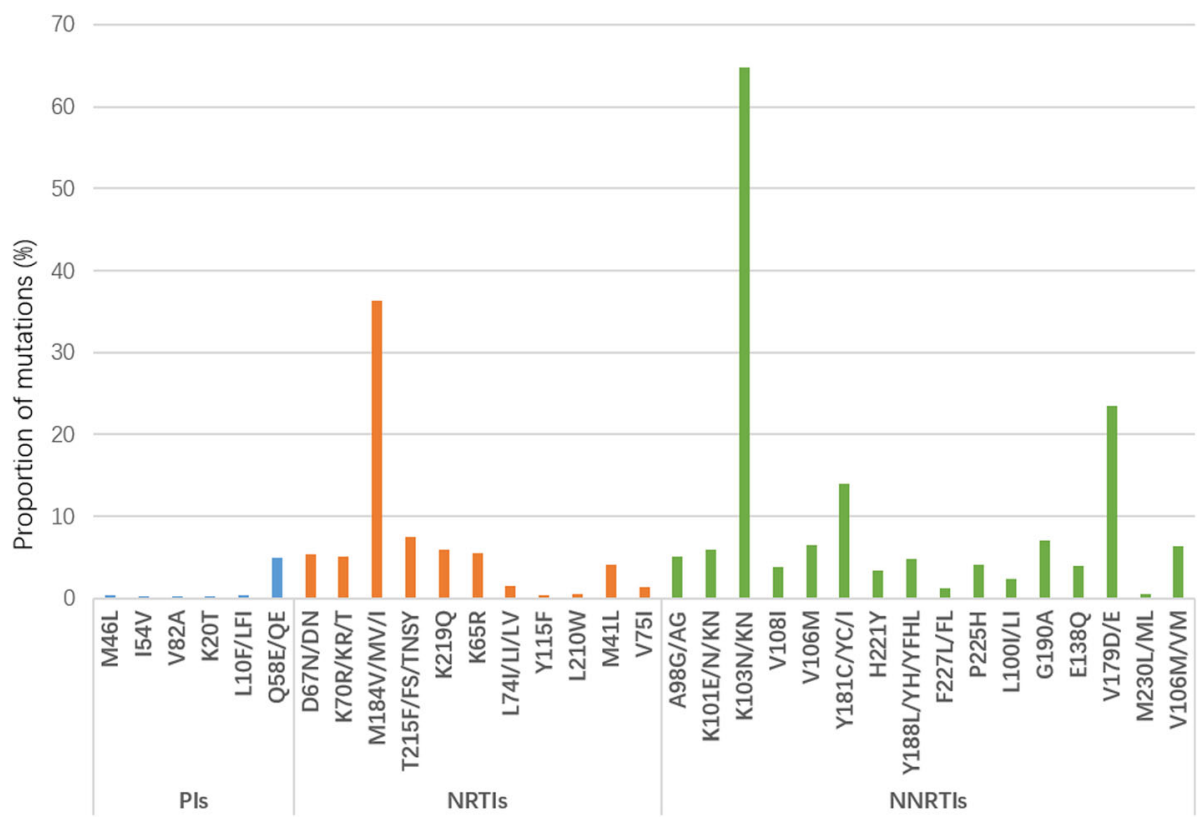

Fig. 2 Drug resistance mutations to PIs, NRTIs and NNRTIs. Note: PIs mutations: M46I, 154V, V82A, K20T, L10F/LFI and Q58E/QE; NRTIs mutations: D67N/DN, K70R/KR/T, M184V/MV/I, T215F/FS/TNSY, K219Q, K65R, L74I/LI/LV, Y115F, L210W, M41L and V75I; NNRTIs mutation: A98G/AG, K101E/N/ KN, K103N/KN, V108I, V106M, Y181C/YC/I, H221Y, Y188L/YH/YFHL, F227L/FL, P225H, L100I/LI, G190A, E138Q, V179D/E, M230L/ML and V106M/VM

According to our findings, drug-resistant frequency to NNRTIs was much higher than that to NRTIs and PIs in this area among PLWH with virologic failure in ART. A similar pattern was also found in other places in China and low- and middle-income countries [43-46], but the prevalence of NNRTI resistance in this area is lower than in other places (50-90\%) [47]. Thus, the high prevalence of virologic failure in ART and related low prevalence of drug resistance may be mainly attributed to poor treatment adherence, and strategies are needed to improve treatment adherence and reduce the treatment failure in ART.

There were several limitations in this study. First, this study was a cross-sectional survey, time-based sequence and cause-effect relationships among these variables cannot be established. Second, some HIV related behaviors were self-reporting, which may lead to recall bias. Third, some important factors of treatment failure in ART, such as ART adherence, should be considered as potential factors in our study, but such information did not collect during the follow-up. Fourth, the participants enrolled in the study were not newly diagnosed with HIV-1 infection, and some patients may have pretreatment drug resistance. Therefore, we could not identify the time of virologic failure and the resistance to ART before and after genotyping in the first virologic failure.

\section{Conclusions}

The findings confirmed that the prevalence of incomplete viral suppression of ART virologic failure in ART among
PLWH was high in this area. On the one hand, we should develop more suitable health education measures for medication compliance for this kind of population; on the other, the early identification of the drug resistance of PLWH with ART virologic failure are of great significance. Timely replacement of new regimens for PLWH with drug resistance could prevent additional drug resistance mutation or multiple drug resistance. Last but not the least, continuum in care and retention in care are important factors in preventing virological failure [48], and enhanced understandings of adherence and adherence interventions for less healthy individuals are required to reduce the virological failure. Also, understanding the genotype distribution and drug resistance may contribute to designing target preventive interventions for improving treatment efficiency, aiming for the selection of more effective therapeutic regimens to promote viral suppression and prevent HIV-1 transmission.

\section{Abbreviations}

HIV: Human immunodeficiency virus; AIDS: Acquired Immune Deficiency Syndrome; ART: Antiretroviral therapy; PLWH: People living with HIV; VCT: Voluntary Counseling and Testing; NGO: Non-government organization; HSS: HIV sentinel surveillance; RT-PCR: Reverse transcription-polymerase chain reaction; OR: Odds ratios; Cl: Confidence interval; NNRTI: Nonnucleoside reverse transcriptase inhibitor; NRTI: Nucleoside reverse transcriptase inhibitor; PI: Protease inhibitor; TDF: Tenofovir; 3TC: Lamivudine; NVP: Nevirapine; EFV: Efavirenz; AZT: Zidovudine; LPV/r: Tipranavir/ritonavir; ATV/r: Atazanavir/ritonavir; DRv/r: Darunavir/ritonavir; FPV/r: Tipranavir/ ritonavir; IDV/r: Indinavir/Ritonavir; NFV: Nelfinavir; SQV/r: Ritonavir-boosted saquinavir; TPV/r: Tipranavir/ritonavir; ABC: Abacavir; D4T: Stavudine; DDI: Didanosine; FTC: Emtricitabine; ETR: Etravirine; RPV: Rilpivirine 


\section{Acknowledgments}

The authors would like to thank the Center for AIDS/STD Control and Prevention, Sichuan Center for Disease Control and Prevention, Liangshan Center for Disease Control and Prevention and Butuo Center for Disease Control and Prevention to support data.

\section{Authors' contributions}

DY, SY and SL had taken a principal role in the conception of ideas, writing the protocol, developing methodologies, analyses and write up of the article and drafted the manuscript. ML contributed to the proposal writing and made a critical revision to the paper and manuscript. PJ contributed to the write up of the study protocol and made revision to the paper. $Y L, Y H, L Y$, $L A, M C, C Y$ and $H L$ supervised the study. ZW made revision to the paper. All authors read and approved the final manuscript.

\section{Funding}

This research was funded by the National Natural Science Foundation of China (81703279); Sichuan Science and Technology Program (2019YJ0148): National Major Science and Technology Projects of China: Study on the assessment technology and prediction model of AIDS epidemic in China (2017ZXX10201101).

\section{Availability of data and materials}

The datasets used and analyzed during the current study are available from the corresponding author on reasonable request.

\section{Ethics approval and consent to participate}

All subjects voluntarily participated in our study and signed informed consent forms before enrollment. For participants under 16 years old, written informed consent was obtained from their parents or guardians. The study protocol was approved by the Ethics Committee of the Sichuan Center for Disease Control and Prevention (201303), and the study was carried out following the Helsinki Declaration of 1964.

\section{Consent for publication}

Not application.

\section{Competing interests}

The authors declare that they have no competing interests.

\section{Author details}

${ }^{1}$ Center for AIDS/STD Control and Prevention, Sichuan Center for Disease Control and Prevention, Chengdu, China. ${ }^{2}$ West China School of Public Health and West China Fourth Hospital, Sichuan University, Chengdu, Sichuan, China. ${ }^{3}$ Department of Land Surveying and Geo-Informatics, The Hong Kong Polytechnic University, Hong Kong, China. International Initiative on Spatial Lifecourse Epidemiology (ISLE), Hong Kong, China. ${ }^{5}$ Butuo Center for Disease Control and Prevention, Butuo, China. ' Liangshan Center for Disease Control and Prevention, Xichang, China. 'Butuo County People's Hospital, Butuo, China. ${ }^{8}$ Centre for Health Behaviours Research, The Jockey Club School of Public Health and Primary Care, The Chinese University of Hong Kong, Hong Kong, China.

\section{Received: 27 January 2020 Accepted: 27 May 2020}

Published online: 23 June 2020

\section{References}

1. UNAIDS. Global AIDS monitoring 2018, country progress report - China: 2018. Available at: http://www.unaids.org/en/dataanalysis/ knowyourresponse/globalaidsprogressreporting/.

2. Yang S, Luo M, Zhang S, Yao Y, Wang Q, Liao Q. Overview on culture and customs related to AIDS epidemic and prevention among Yi people in Liangshan. Chin J AIDS STD. 2017;23(3):101-5.

3. Zhao QG, Gong YH, Liao Q, Yu G, Wang K, Wang J, et al. Estimation on the HIV-1 incidence in Liangshan Yi autonomous prefecture, under BED-capture enzyme immunoassay, from 2011 to 2013. Zhonghua Liu Xing Bing Xue Za Zhi. 2016;37(8):1105-7.

4. Sun X, Lu F, Wu Z, Poundstone K, Zeng G, Xu P, et al. Evolution of information-driven HIV/AIDS policies in China. Int J Epidemiol. 2010;1(Suppl 2):ii4-13.
5. Günthard HF, Aberg JA, Eron JJ, Hoy JF, Telenti A, Benson CA, et al. Antiretroviral treatment of adult HIV infection: 2014 recommendations of the international antiviral society-USA panel. JAMA. 2014;312(4):410-25.

6. Cohen MS, Chen YQ, McCauley M, Gamble T, Hosseinipour MC, Kumarasamy $\mathrm{N}$, et al. Prevention of HIV-1 infection with early antiretroviral therapy. N Engl J Med. 2011;365(6):493-505.

7. Beyrer C, Pozniak A. HIV drug resistance - an emerging threat to epidemic control. N Engl J Med. 2017;377(17):1605-7.

8. Zhu H, Napravnik S, Eron JJ, Cole SR, Ma Y, Wohl DA, et al. Decreasing excess mortality of HIV-infected patients initiating antiretroviral therapy: comparison with mortality in general population in China, 2003-2009. J Acquir Immune Defic Syndr. 2013;63(5):e150-7.

9. Sanguansittianant S, Nooroon N, Phaengchomduan P, Ammaranond P. Trends in prevalence of HIV-1 drug resistance in Thailand 2009-2010. J Clin Lab Anal. 2013;27(5):346-53.

10. Yang $C B$, Yang W, Zeng YL, Gu XD, Bian SC, Wu M, et al. Effect of early antiretroviral therapy on acquired immune deficiency syndrome in Butuo County, Liangshan autonomous prefecture. West China Med J. 2017;32(6): 867-70.

11. Xu JL, Jike KCN, Ma Y, Yu G, Wang J, Wang K, et al. Effects and associated factors of HIV/AIDS anti-retroviral therapy in Liangshan Yi autonomous refecture, Sichuan Province. Zhonghua Yu Fang Yi Xue Za Zhi. 2018;52(6): 668-72.

12. Dou Z, Zhang F, Zhao Y, Jin C, Zhao D, Gan X, et al. Progress on China s national free antiretroviral therapy strategy in 2002-2014. Zhonghua Liu Xing Bing Xue Za Zhi. 2015;36(12):1345-50.

13. Clutter DS, Jordan MR, Bertagnolio S, Shafer RW. HIV-1 drug resistance and resistance testing. Infect Genet Evol. 2016;46:292-307.

14. Castro H, Pillay D, Cane P, Asboe D, Cambiano V, Phillips A, et al. Resistance. UCGoHD: persistence of HIV-1 transmitted drug resistance mutations. J Infect Dis. 2013;208(9):1459-63.

15. Leigh Brown AJ, Lycett SJ, Weinert L, Hughes GJ, Fearnhill E, Dunn DT. Collaboration. UHDR: transmission network parameters estimated from HIV sequences for a nationwide epidemic. J Infect Dis. 2011;204(9):1463-9.

16. Hemelaar J. Implications of HIV diversity for the HIV-1 pandemic. J Inf Secur. 2013;66(5):391-400.

17. Song YX, Xin RL, Li ZC, Yu HW, Lun WH, Ye J, et al. Prevalence of transmitted drug resistance among HIV-1 treatment-naive patients in Beijing. Epidemiol Infect. 2018;146(3):339-44.

18. Xiao P, Zhou Y, Lu J, Yan L, Xu X, Hu H, et al. HIV-1 genotype diversity and distribution characteristics among heterosexually transmitted population in Jiangsu province, China. Virol J. 2019;16(1):51.

19. Li X, Li W, Zhong P, Fang K, Zhu K, Musa TH, et al. Nationwide trends in molecular epidemiology of HIV-1 in China. AIDS Res Hum Retrovir. 2016; 32(9):851-9

20. Borghetti A, Moschese D, Cingolani A, Baldin G, Speziale D, Ciccullo A, et al. Lamivudine-based maintenance antiretroviral therapies in patients living with HIV-1 with suppressed HIV RNA: derivation of a predictive score for virological failure. HIV Med. 2019;20(9):624-7.

21. Amstutz A, Nsakala BL, Vanobberghen F, Muhairwe J, Glass TR, Achieng B, et al. SESOTHO trial ("switch either near suppression or THOusand") - switch to second-line versus WHO-guided standard of care for unsuppressed patients on first-line ART with viremia below 1000 copies $/ \mathrm{mL}$ : protocol of a multicenter, parallel-group, open-label, randomized clinical trial in Lesotho, southern Africa. BMC Infect Dis. 2018:18(1):76.

22. Yuan D, Su L, Liu H, Yu H, Yang H, Ye L, et al. Drug-resistance characteristics of CRF01_AE and CRF07_BC subtypes of HIV-1 strains in Sichuan province. Zhonghua Yu Fang Yi Xue Za Zhi. 2015;49(10):901-6.

23. Yuan D, Du Z, Zhou J, Ye L, Su L, Yang H, et al. HIV-1 subtype diversity, drug resistance, and genetic transmission networks in men who have sex with men with virologic failure in antiretroviral therapy in Sichuan, China, 2011 to 2017. Medicine (Baltimore). 2019;98(43):e17585.

24. Xiaoli C, Yun L, Junbin L, Fengyu H, Weiping C. Analysis on drug resistance in HIV/AIDS patients with HAART through different infection routes in Guangdong. Chin J AIDS STD. 2015;21(5):369-72.

25. Maojiao L. Analysis of genotype resistance variation and subtypes of HIV-1 in Honghe prefecture, Yunnan. Dali: Dali University; 2018.

26. Bulage L, Ssewanyana I, Nankabirwa V, Nsubuga F, Kihembo C, Pande G, et al. Factors associated with Virological non-suppression among HIVpositive patients on antiretroviral therapy in Uganda, august 2014-July 2015. BMC Infect Dis. 2017;17(1):326. 
27. Gupta A, Wood R, Kaplan R, Bekker LG, Lawn SD. Prevalent and incident tuberculosis are independent risk factors for mortality among patients accessing antiretroviral therapy in South Africa. PLoS One. 2013;8(2):e55824.

28. Tran DA, Wilson DP, Shakeshaft A, Ngo AD, Doran C, Zhang L. Determinants of virological failure after 1 year's antiretroviral therapy in Vietnamese people with HIV: findings from a retrospective cohort of 13 outpatient clinics in six provinces. Sex Transm Infect. 2014;90(7):538-44.

29. Jordan MR, Obeng-Aduasare Y, Sheehan H, Hong SY, Terrin N, Duong DV, et al. Correlates of non-adherence to antiretroviral therapy in a cohort of HIV-positive drug users receiving antiretroviral therapy in Hanoi, Vietnam. Int J STD AIDS. 2014;25(9):662-8

30. Cauldbeck MB, O'Connor C, O'Connor MB, Saunders JA, Rao B, Mallesh VG, et al. Adherence to anti-retroviral therapy among HIV patients in Bangalore, India. AIDS Res Ther. 2009;6:7

31. Chawana TD, Reid A, Bwakura T, Gavi S, Nhachi CF. Factors influencing treatment failure in HIV positive adult patients on first line antiretroviral therapy. Cent Afr J Med. 2014;60(5-8):29-36.

32. Jean Louis F, Buteau J, Francois K, Hulland E, Domercant JW, Yang C, et al. Virologic outcome among patients receiving antiretroviral therapy at five hospitals in Haiti. PLoS One. 2018;13(1):e0192077.

33. Kyaw NT, Harries AD, Kumar AM, Oo MM, Kyaw KW, Win T, et al. High rate of virological failure and low rate of switching to second-line treatment among adolescents and adults living with HIV on first-line ART in Myanmar, 2005-2015. PLoS One. 2017;12(2):e0171780.

34. Yu X-F, Chen J, Shao Y, Beyrer C, Lai S. Two subtypes of HIV-1 among injection-drug users in southern China. Lancet. 1998;351:9111.

35. Yu ES, Xie Q, Zhang K, Lu P, Chan LL. HIV infection and AIDS in China, 1985 through 1994. Am J Public Health. 1996;86(8):1116-22.

36. Lin YT, Lan YC, Chen YJ, Huang YH, Lee CM, Liu TT, et al. Molecular epidemiology of HIV-1 infection and full-length genomic analysis of circulating recombinant form 07_BC strains from injection drug users in Taiwan. J Infect Dis. 2007;195(9):1283-93.

37. He X, Xing H, Ruan Y, Hong K, Cheng C, Hu Y, et al. A comprehensive mapping of HIV-1 genotypes in various risk groups and regions across China based on a nationwide molecular epidemiologic survey. PLoS One. 2012;7(10):e47289.

38. Su L, Graf M, Zhang Y, von Briesen H, Xing H, Köstler J, et al. Characterization of a virtually full-length human immunodeficiency virus type 1 genome of a prevalent intersubtype $\left(C / B^{\prime}\right)$ recombinant strain in China. J Virol. 2000;74(23):11367-76.

39. Piyasirisilp S, McCutchan FE, Carr JK, Sanders-Buell E, Liu W, Chen J, et al. A recent outbreak of human immunodeficiency virus type 1 infection in southern China was initiated by two highly homogeneous, geographically separated strains, circulating recombinant form $A E$ and a novel $B C$ recombinant. J Virol. 2000;74(23):11286-95.

40. Tee KK, Pybus OG, Li XJ, Han X, Shang H, Kamarulzaman A, et al. Temporal and spatial dynamics of human immunodeficiency virus type 1 circulating recombinant forms 08_BC and 07_BC in Asia. J Virol. 2008;82(18):9206-15.

41. Zhefeng $M$, Jingyun $L$, Ping $Z$, Jianqing $X$, Xiaoyan Z. A new transmission map of HIV-1 CRF07_BC in China: analysis of sequences from 12 provinces over a decade. Retrovirology. 2012;9(S2):P139.

42. Qixing W, Suhua Z, Shujuan Y, Qiang L, Gang Y, Yongnan Y, et al. Characteristics of transmission route among the reported HIV infections during 2011-2014 in Liangshan prefecture. Chin J AIDS STD. 2017;23(8):71922

43. Hou LJ, Wang HW, Duan SP, Zhuo Y, Zhou YC, Wu HJ, et al. The prevalence and determinants of drug-resistance-associated mutations in the HIV-1infected MSM population of Henan Province in China. Arch Virol. 2015; 160(8):2051-61.

44. lyidogan P, Anderson KS. Current perspectives on HIV-1 antiretroviral drug resistance. Viruses. 2014;6(10):4095-139.

45. Menéndez-Arias L. Targeting HIV: antiretroviral therapy and development of drug resistance. Trends Pharmacol Sci. 2002;23(8):381-8.

46. Cunningham E, Chan YT, Aghaizu A, Bibby DF, Murphy G, Tosswill J, et al. Enhanced surveillance of HIV-1 drug resistance in recently infected MSM in the UK. J Antimicrob Chemother. 2017;72(1):227-34.

47. WHO. HIV drug resistance report 2017. Geneva: World Health Organization; 2017

48. Nsanzimana S, Semakula M, Ndahindwa V, Remera E, Sebuhoro D, Uwizihiwe JP, et al. Retention in care and virological failure among adult
HIV+ patients on second-line ART in Rwanda: a national representative study. BMC Infect Dis. 2019;19(1):312.

\section{Publisher's Note}

Springer Nature remains neutral with regard to jurisdictional claims in published maps and institutional affiliations.

\section{Ready to submit your research? Choose BMC and benefit from:}

- fast, convenient online submission

- thorough peer review by experienced researchers in your field

- rapid publication on acceptance

- support for research data, including large and complex data types

- gold Open Access which fosters wider collaboration and increased citations

- maximum visibility for your research: over $100 \mathrm{M}$ website views per year

At BMC, research is always in progress.

Learn more biomedcentral.com/submissions 